\title{
Asteroseismic signatures of magnetic activity variations in solar-type stars
}

\author{
Travis S. Metcalfe ${ }^{1,2}$ \\ ${ }^{1}$ Space Science Institute, Boulder CO 80301 USA; \\ email: travis@spsci.org \\ ${ }^{2}$ Stellar Astrophysics Centre, Aarhus University, DK-8000 Aarhus C, Denmark
}

\begin{abstract}
Observations of magnetic activity cycles in other stars provide a broader context for our understanding of the 11-year sunspot cycle. The discovery of short activity cycles in a few stars, and the recognition of analogous variability in the Sun, suggest that there may be two distinct dynamos operating in different regions of the interior. Consequently, there is a natural link between studies of magnetic activity and asteroseismology, which can characterize some of the internal properties that are relevant to dynamos. I provide a brief historical overview of the connection between these two fields (including prescient work by Wojtek Dziembowski in 2007), and I highlight some exciting results that are beginning to emerge from the Kepler mission.
\end{abstract}

Keywords. stars: activity, stars: interiors, stars: magnetic fields, stars: oscillations

\section{Background}

In early 2005, I attended a seminar given by David Salabert in which he described his work documenting subtle shifts in the solar oscillation frequencies throughout the 11-year sunspot cycle (Salabert et al. 2004). The measurements relied on data from the IRIS network, and they clearly showed that even the low-degree $(l \leqslant 3)$ solar oscillation frequencies were shifted by a few tenths of a $\mu \mathrm{Hz}$ between magnetic minimum and maximum. If such frequency shifts were detectable in the Sun observed as a star, I realized that it might be possible to see similar behavior in other stars.

The high-degree oscillation frequency shifts in the Sun through the solar cycle were first characterized by Libbrecht \& Woodard (1990). Using the thousands of oscillation modes then available from helioseismology they showed that the magnitude of the shift depended on both the geometry (spherical degree, $l$ ) and the frequency of the oscillation, with the largest shifts observed for higher degrees and at higher frequencies. The initial interpretation of these observations was given by Goldreich et al. (1991), who matched the frequency dependence of the shifts by considering a direct magnetic perturbation to the near-surface propagation speed of the acoustic waves.

Dziembowski \& Goode (2005) developed a similar formalism to explain modern spacebased observations of the solar acoustic oscillations ( $p$ modes) as well as the surface gravity waves (f modes). They identified some secondary effects that were needed to explain the shifts observed in both sets of modes: a decrease in the radial component of the turbulent velocity, and the associated changes in temperature. Shortly after this work was published, I contacted Wojtek Dziembowski to ask whether I could use his code to calculate the expected shifts in low-degree p modes for other solar-type stars.

At the time there were very few stars with detections of solar-like oscillations, but Fletcher et al. (2006) would soon publish evidence of a marginally significant shift in the p-mode frequencies of $\alpha$ Cen A by comparing ground-based observations with earlier data from the WIRE satellite, and Bedding et al. (2007) would see a similar (but statistically 
insignificant) shift when comparing two ground-based asteroseismology campaigns on $\beta$ Hyi. Wojtek happily sent me a copy of his code, and with his help I spent more than a year trying to figure out how to adapt it for other stars before he generously invited me to come to Warsaw for a week and work on it together. During that week we kick-started a project on $\beta$ Hyi, and we submitted the paper six months later (Metcalfe et al. 2007).

\section{Predictions}

As the activity level of the Sun rises from minimum to maximum, the p-mode oscillations are gradually shifted to higher frequencies. The magnitude of the shift is proportional to the change in activity level, so the simplest prediction for other stars is to assume that the mean shift in the p-mode frequencies scales with activity level. In other words, the largest shifts would be expected in the most active stars. This was the approach taken by Chaplin et al. (2007), who were working contemporaneously.

Wojtek took a slightly different approach to predicting the frequency shifts in other stars. He parametrized the shifts as $\Delta \nu \propto A_{0}[R / M] Q_{j}\left(D_{c}\right)$, where $A_{0}$ scales with the activity level as in Chaplin et al. (2007), $R$ and $M$ are the radius and mass, and $Q_{j}$ is a function of $D_{c}$ which is the depth of the source of the perturbation below the photosphere. He demonstrated that this parametrization could remove all of the dependence on spherical degree and most of the frequency dependence in MDI data of the Sun with $D_{c}$ fixed at $0.3 \mathrm{Mm}$. To extend the relation to other stars, he assumed that $D_{c}$ would scale with the pressure scale height $H_{p}$ in the outer layers: $D_{c} \propto H_{p} \propto L^{1 / 4} R^{3 / 2} / M$, which can be expressed in terms of the luminosity $L$, radius and mass.

At the time we were doing this work, $\beta$ Hyi was the only star with a known activity cycle and multiple asteroseismic observing campaigns. The activity cycle had been observed in the Mg II h and $\mathrm{k}$ lines by the IUE satellite, and several years of additional observations were available in the archive after the initial characterization by Dravins et al. (1993). Phil Judge did a complete reanalysis of the IUE data, and determined a cycle period of 12 years with a maximum at 1986.9. Marty Snow produced a comparable record of solar $\mathrm{Mg}$ II $\mathrm{h}$ and $\mathrm{k}$ flux so we could scale the observed change in $\beta$ Hyi to predict a mean shift in the oscillation frequencies. Just by luck, $\beta$ Hyi was near magnetic maximum during the first detection of solar-like oscillations by Bedding et al. (2001), and close to magnetic minimum during the subsequent asteroseismic campaign by Bedding et al. (2007). The ground-based data were insufficient for a quantitative test, but Wojtek's relation qualitatively reproduced the observed shifts (Metcalfe et al. 2007).

Two years later, Christoffer Karoff led a project to define an optimal sample of asteroseismic targets in the Kepler field that would also be monitored for stellar activity variations from ground-based $\mathrm{Ca}$ II $\mathrm{H}$ and $\mathrm{K}$ measurements obtained throughout the mission (Karoff et al. 2009, 2013). When trying to determine which stars would show the largest frequency shifts, he was confronted with two conflicting predictions (see Fig. 1). The relation proposed by Chaplin et al. (2007) suggested that the largest shifts would be expected in the young active K stars. Wojtek's relation in Metcalfe et al. (2007) predicted that the hotter F stars would exhibit the largest shifts, which would grow even larger as the stars evolved. To be safe, the sample covered the full range of temperatures.

\section{Confirmation}

At the Beijing SONG workshop in March 2010, I gave a contributed talk on monitoring stellar magnetic activity cycles with SONG. During the coffee break Rafa García told me about some asteroseismic observations of the F star HD 49933, and he invited me to participate. The activity level of the star was not known, so I added it to a time-domain 


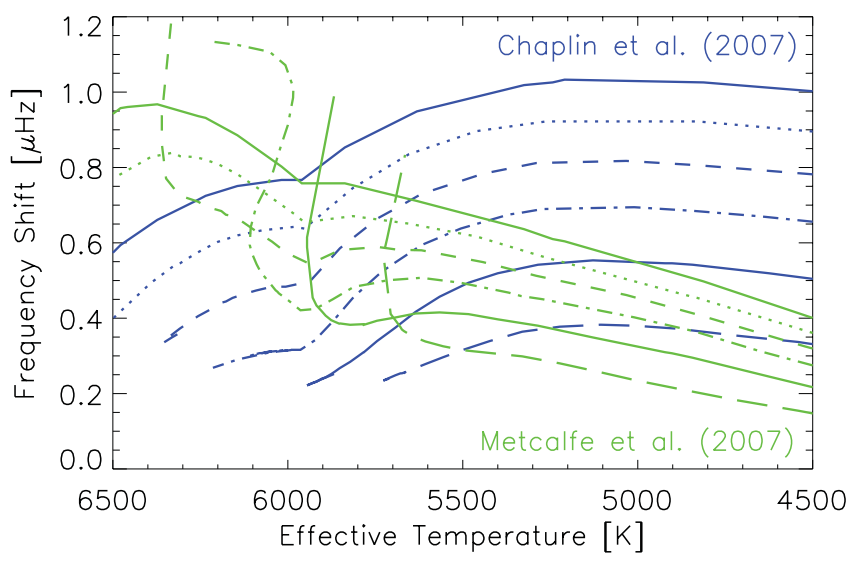

Figure 1. Predictions of the cycle-induced frequency shift as a function of effective temperature and age, with the scaling proposed by Chaplin et al. (2007) shown in blue and that proposed by Metcalfe et al. (2007) shown in green. Solid lines are Padova isochrones at an age of 1 Gyr, and the sets of dashed lines show progressively older isochrones at 1.6, 2.5, 4, 6.3, and $10 \mathrm{Gyr}$ (adapted from Karoff et al. 2009).

survey of $\mathrm{Ca}$ II $\mathrm{H}$ and $\mathrm{K}$ emission that I was conducting for a sample of bright stars in the southern hemisphere (Metcalfe et al. 2009).

García et al. (2010) discovered anti-correlated changes in the frequencies and amplitudes of the oscillations in HD 49933 during 150 days of continuous monitoring by CoRoT. The frequency shifts were positive, and passed through a minimum while the amplitudes increased and passed through a maximum - the same pattern of changes that occurs in the Sun as it passes through a magnetic minimum. Convection stochastically excites and intrinsically damps solar-like oscillations. Magnetic fields inhibit convection, suppressing the oscillation amplitudes while simultaneously shifting the frequencies.

Salabert et al. (2011) pushed the analysis further, and examined the frequency dependence of the shifts. Just as in the Sun, the shifts grew steadily larger toward higher frequencies. It seemed clear that CoRoT had made the first asteroseismic detection of a stellar magnetic cycle, but there was one striking difference between HD 49933 and the Sun. The frequency shifts observed in this $\sim 2$ Gyr-old F star at $6600 \mathrm{~K}$ were $4-5$ times larger than solar, providing the first confirmation of Wojtek's relation.

\section{Future prospects}

The archive of Kepler data represents an unprecedented opportunity to study the short-period magnetic cycles that have been observed in some rapidly rotating $\mathrm{F}$ stars. The high precision time-series photometry collected every 30 minutes over the past four years can be used to measure rotation periods from spot modulation and to monitor the longer-term brightness changes associated with the stellar cycle. Furthermore, for targets that have been observed in short cadence (1-minute sampling), asteroseismology allows a characterization of the star including key dynamo ingredients such as the depth of the surface convection zone (Mazumdar et al. 2012) and radial differential rotation (Deheuvels et al. 2012). The asteroseismic data can also be used to monitor the solar-like oscillations over time, allowing a search for the same pattern of changes that have been seen for the Sun and HD 49933 in response to their magnetic cycles. Rapidly rotating F stars are the ideal targets because they show the shortest cycle periods (Metcalfe et al. 2010), and the frequency shifts are significantly larger than in the Sun (see Fig. 1). Young, 
rapidly rotating $\mathrm{K}$ stars can also show relatively short cycles (Metcalfe et al. 2013), but the asteroseismic signatures are expected to be smaller.

Mathur et al. (2013) recently examined the archive of Kepler observations for a sample of 22 rapidly rotating $\mathrm{F}$ stars. Wavelet analysis of the long light curves revealed clear signatures of latitudinal differential rotation and evidence for short magnetic cycles in a few stars. The best target in the sample has three years of continuous asteroseismic data spanning what appears to be a complete magnetic cycle, so additional tests of Wojtek's relation should soon be possible.

\section{Acknowledgements}

This long story has gradually unfolded with the collaboration and support of Tim Bedding, Phil Judge, Christoffer Karoff, Bill Chaplin, Rafa García, Savita Mathur, and David Salabert. I would particularly like to thank Wojtek Dziembowski for graciously inviting me to work with him at the Copernicus Astronomical Center for a week in September 2006. This work was partially supported by NASA grant NNX13AC44G.

\section{References}

Bedding, T. R., Butler, R. P., Kjeldsen, H., et al. 2001, ApJ, 549, L105

Bedding, T. R., Kjeldsen, H., Arentoft, T., et al. 2007, ApJ, 663, 1315

Chaplin, W. J., Elsworth, Y., Houdek, G., \& New, R. 2007, MNRAS, 377, 17

Deheuvels, S., García, R. A., Chaplin, W. J., et al. 2012, ApJ, 756, 19

Dravins, D., Linde, P., Fredga, K., \& Gahm, G. F. 1993, ApJ, 403, 396

Dziembowski, W. A. \& Goode, P. R. 2005, ApJ, 625, 548

Fletcher, S. T. Chaplin, W. J., Elsworth Y., et al. 2006, MNRAS, 371, 935

García, R. A., Mathur, S., Salabert, D., et al. 2010, Science, 329, 1032

Goldreich, P., Murray, N., Willette, G., \& Kumar, P. 1991, ApJ, 370, 752

Karoff, C., Metcalfe, T. S., Chaplin, W. J., et al. 2009, MNRAS, 399, 914

Karoff, C., Metcalfe, T. S., Chaplin, W. J., et al. 2013, MNRAS, 433, 3227

Libbrecht, K. G. \& Woodard, M. F. 1990, Nature, 345, 779

Mathur, S., García, R. A., Ballot, J., et al. 2013, A\&A, submitted

Mazumdar, A., Monteiro, M. J. P. F. G., Ballot, J., et al. 2012, AN, 333, 1040

Metcalfe, T. S., Dziembowski, W. A., Judge, P. G., \& Snow, M. 2007, MNRAS, 379, L16

Metcalfe, T. S., Judge, P. G., Basu, S., et al. 2009, Solar Analogs II workshop (arXiv: 0909.5464)

Metcalfe, T. S., Basu, S., Henry, T. J., et al. 2010, ApJ, 723, L213

Metcalfe, T. S., Buccino, A. P., Brown, B. P., et al. 2013, ApJ, 763, L26

Salabert, D., Fossat, E., Gelly, B., et al. 2004, A\&\&A, 413, 1135

Salabert, D., Régulo, C., Ballot, J., et al. 2011, A\&SA, 530, A127 